ROCZNIKI TEOLOGICZNE

Tom LXVI, zeszyt 5 - 2019

DOI: http://dx.doi.org/10.18290/rt.2019.66.5-4

LLUÍS CLAVELL

\title{
L’UNIVERSITÀ AL SERVIZIO DELL’UOMO INTEGRALE
}

\author{
UNIVERSITY OF INTEGRAL HUMAN SERVICE
}

\begin{abstract}
The author begins the article by giving two examples on the subject of professional work done with excellent preparation and enabling the provision of assistance to a person in his personal and social dimension. He is in favor of a university model that acquires and transfers knowledge in the service of man and society. This should be done by the cooperation of various scientific disciplines at the university and the preparation of the scientific staff should be focused on reliable scientific research and appropriate didactic message.
\end{abstract}

Key words: Universit; scientific research; didactics; service; integral man.

\section{UN RADIOLOGO GIAPPONESE E UN ECONOMISTA ITALIANO}

Nel 1997 è apparsa la prima edizione polacca del libro $A$ song for Nagasaki (1988), tradotto in diverse lingue. In esso l'australiano Paul Glynn racconta la storia del Dottore Takashi Nagai, radiologo e scrittore influente in Giappone, in modo particolare dopo il bombardamento nucleare di Nagasaki. Nagai si prepara per ottenere la laurea in medicina con un grande desierio di servire ma anche con una visione dell'uomo, come semplice organismo biologico. A metà dei suoi studi, sua mamma soffre un attacco di apoplessia. Avvertito dal padre, lui prende subito il treno. Arriva e trova mamma cosciente, ma senza poter parlare. Lo sguardo affettuoso della mamma negli ultimi dieci minuti di vita fanno intuire a Takashi che, mamma non è solo organismo materiale: esiste lo spirito umano che vive oltre la morte. Il suo riduzionismo è caduto di colpo. Ma adesso bisogna riflettere su questa intuizione. Grande lettore, ritorna ai pensieri di Pascal, che ha portato con sé

LLuís CLAVELL - rofessore di metafisica in pensione, Facoltà di filosofia, Pontificia Università della Santa Croce, Roma, Italia; Indirizzo di corrispondenza: Piazza di Sant'Apolinare 49; 00186 Roma; e-mail: 1luisclavell@gmail.com 
nel viaggio a casa della madre. Torna ai suoi studi e, senza avere lui ancora una risposta, incomincia a mettere in difficoltà i professori che offrono spiegazioni materialistiche e trattano con freddezza i pazienti.

Trascorre cinque anni con la domanda sul senso della vita. La lettura di Pascal lo porta a conoscere i cristiani e si converte a Cristo. Un problema acustico gli impedisce di esercitare come medico e si dedica alla radiologia, consapevole dell'alto rischio di contrarre la leucemia a causa della scarsa protezione allora dai raggi X. Sposa una donna cattolica, soffre la tragedia di Nagasaki e aiuta i giapponesi ad accettare la Provvidenza divina, che ha permesso questi sacrificio come mezzo per la pace. Nel 2015 le Edizioni Paoline hanno pubblicato il libro in italiano con il suggestivo titolo: Pace su Nagasaki. Il medico che guariva i cuori.

Ho voluto iniziare cosí il mio contributo L'università al servizio dell' uomo integrale al fascicolo della rivista Roczniki Teologiczne dedicato alla spiritualità dell'università, ed è stato oggetto alla XL EDIZIONE MEETING PER L'AMICIZIA FRA I POPOLI di Rimini di 2019 di una mostra "Takashi Paolo Nagai. Annuncio da Nagasaki" proposta da Medicina e Persona, perché mi sembra un esempio di un universitario profondo con un grande spirito di servizio legato alla ricerca della verità. Ci sarebbero molti altri professori nel campo della medicina, come ad esempio, Jérôme Lejeune (1926-1994) genetista francese, scopritore della causa della sindrome di Down, e primo presidente della Pontificia accademia per la vita, creata da San Giovanni Paolo II.

Ma vorrei anche menzionare un altro universitario, questa volta nell'ambito dell'economia: Giuseppe Toniolo (1845-1918), ordinario di economia politica a Pisa, sposato e padre di sette figli, promotore delle Settimane sociali dei cattolici italiani, grande collaboratore di Leone XIII e San Pio X. Agostino Gemelli si rivolge a lui perché lo aiuti a costituire in Italia una Università Cattolica.Toniolo vi partecipa come profondo conoscitore degli ordinamenti universitari europei e crede nella necessità di incentivare un sempre più alto livello di studi da parte dei cattolici. La sua idea di università cattolica rimane di grande attualità.

Nel suo Trattato di Economia Sociale propone una concezione etico-giuridica che cerca un'originale integrazione dell'utile individuale con il bene comune, un capitale al servizio del lavoro. Rifiuta la visione utilitaristico-individualista dell'agente economico e sottolinea la complementarità dell'economia con altre discipline sociali, guardando l'economia come scienza dei mezzi ai fini che si vuole raggiungere. L'impatto del suo pensiero si trova nella Dottrina sociale della Chiesa che Toniolo contribuisce a formulare 
a partire dalla stessa Rerum novarum. Per le campagne, consiglia la diffusione della piccola proprietà e la ricomposizione di una parte dei patrimoni collettivi da sfruttare a beneficio del popolo. Per le città, propone la partecipazione dei lavoratori agli utili dell'azienda con l'abolizione del salariato, propugnando una visione dei rapporti all'interno dell'impresa che, si rivela oggi di grande modernità. Nella proprietà industriale è necessario ricongiungere direttamente il capitalista all'imprenditore e poi l'imprenditore agli operai. Nel 1971 San Paolo VI firma il decreto di eroicità delle virtù, che lo rende venerabile. Nel 2012 è stato beatificato.

Sono due ottimi esempi, tra tanti altri, del lavoro professionale esercitato con seria preparazione universitaria, che permette di aiutare allo sviluppo dell'uomo nella sua integrità personale e sociale.

\section{IL MOTTO DI UNA GIOVANE UNIVERSITA: SAPERE PER SERVIRE}

Università molto celebri hanno titoli o motti che esprimono bene la natura di questa istituzione così importante per l'umanità. Alcuni esempi: l'università più antica di Roma creata da un Papa si chiama „La Sapienza”; quella di Oxford ha come motto nel suo stemma „Dominus illuminatio mea”, mentre quella di Parigi: „Hic et ubique terrarum”. Oltre oceano troviamo „Veritas” a Harvard e „Lux et veritas” a Yale. In Svezia, a Upsala il motto è „Gratiae veritas naturae", commentata così da San Giovanni Paolo II nella sua visita del 1989: la verità della grazia è anche la verità della natura; aggiungo che fa una piacevole impressione leggere al di sopra del portale dell'aula magna, queste parole dell'umanista Thorild che non ho dimenticato: „è bene pensare liberamente, ma pensare correttamente è meglio".

L'università in cui ho lavorato più a lungo non ha un motto, ma ha una sigillo, che vuole rispecchiare bene il suo compito di pontificia e romana. $\mathrm{Si}$ tratta di un cerchio, al cui centro c'è una croce greca con punte di freccia agli estremi, che esprime l'irradiazione della Croce di Cristo in tutte le direzioni, verso tutti i popoli. Nei quadranti che rimangono tra i bracci della croce e il cerchio, ci sono quattro parole: Iesus - Christus - Deus - Homo. Una sintesi che san Josemaría disegnava spesso nei suoi scritti fin da giovane sacerdote per esprimere il suo desiderio di mettere Gesù nei cuori di tutte le persone, di propiziare l'incontro di ogni anima con Cristo, perfectus Deus e perfectus homo. 
In continuità con questa lunga tradizione, mi ha colpito il motto della giovane Universidad del Istmo, nella capitale del Guatemala, un progetto iniziato soprattutto da donne unversitarie: ,sapere per servire”. A mio avviso, alla luce di queste parole si capiscono meglio due caratteristiche delle università. Una più recente è quella di cercare di autopresentarsi come centri di eccellenza, da molti intesa come sinonimo di prestigio nel ranking e nelle valutazioni internazionali e di preparazione seria per trovare presto un lavoro di alto livello. In realtà però sembra giusto e umano ricordare che deve essere eccellenza nel servizio.

La altra caratteristica, più conosciuta e immediata, è quella di comunità accdemiche in cui si cerca di conoscere la Verità e di trasmetterla. Ricerca scientifica e docenza, attività inseparabili, perché senza ricerca non c'è in senso proprio università. Ma il fine, -causa delle cause, secondo Aristotele-, è quello di servire le persone, come hanno fatto i due universitari presentati all'inizio, che vivevano il lavoro con spirito cristiano.

Non a caso la Costituzione apostolica di San Giovanni Paolo II sulle università d'ispirazione cattolica ha come titolo Ex corde Ecclesiae. La Chiesa, come Corpo mistico di Cristo, come famiglia dei discepoli di Gesù e figli di Dio, avendo come commandamento primo quello dell'amore a Dio e agli altri, ha dato luogo a un gran numero d'iniziative di servizio agli altri di ogni tipo. Tra queste un posto importante spetta alle università. San Giovanni Paolo II espresse in modo autobiografico questi ideali e possano queste parole spronare a far progredire le università: „Per lunghi anni io stesso ho fatto una benefica esperienza, che mi ha interiormente arricchito, di ciò che è proprio della vita universitaria: l'ardente ricerca della verità e la sua trasmissione disinteressata ai giovani e a tutti coloro che imparano a ragionare con rigore, per agire con rettitudine e servire meglio la società umana"1.

Papa Francesco costata già nel 2013 in termini molto decisi che „ogni epoca della storia porta in sé elementi critici, ma, almeno negli ultimi quattro secoli, non si sono viste così scosse le certezze fondamentali che costituiscono la vita degli esseri umani come nella nostra epoca [...] E' un cambiamento che riguarda il modo stesso in cui l'umanità porta avanti la sua esistenza nel mondo"2. E nel 2015 nel Duomo di Firenze: „La società italiana si costruisce quando le sue diverse ricchezze culturali possono dialogare in modo costruttivo: quella popolare, quella accademica, quella giovanile,

\footnotetext{
${ }^{1}$ San Giovanni Paolo II, Cost. ap. Ex corde Eclesice, 15 agosto 1990, n. 2.

${ }^{2}$ Francesco, Discorso nella Pontificia Facoltà di Teologia della Sardegna, Cagliari, 22 settembre 2013.
} 
quella artistica, quella tecnologica, quella economica, quella politica, quella dei media..."3. Francesco considera la crisi dell'Europa molto grave perché ne va di mezzo la cultura cristiana, come ricordava in occasione del premio Carlomagno. Chiede anche di aiutare i laici in modo non clericale: facilitare formazione per il loro lavoro e la loro famiglia, e incoraggiare la loro partecipazione nella vita pubblica ${ }^{4}$.

L'idea di sapere per servire pervade l'Enciclica Laudato si' con cui il Romano Pontefice vuole rilanciare la Dottrina sociale della Chiesa. Uno dei punti più importanti è il superamento della frammentazione, per avere una visione integrale, che poi permetta di agire in profondità. Papa Francesco esprime il suo realismo con alcune formule che ormai conosciamo: $L a$ realtà è più importante dell'idea; Il tutto è superiore alla parte. Queste due mi sembrano particolarmente adeguate al tema, perché hanno una ricaduta nel lavoro universitario, il quale, secondo me, soffre per la pressione verso una specializzazione formativa troppo veloce (addiritura fin dal liceo), a cui consegue una tendenza a far diventare le facoltà alte scuole professionali. Questo profilo non facilita cogliere il tutto e rende difficile una cultura capace di andare oltre ai riduzionismi economicista e scientistico e di superare il ruolo molto forte dello stato sia nazionale che europeo.

\section{L'INTERDISCIPLINARIETA A SERVIZIO DELL'UOMO INTEGRALE}

La altissima perfezione dell'essere umano e di tutta la creazione ha causato una cultura molto ricca e ha fatto nascere numerose scienze e moltissimi tipi di lavori professionali che riguardano la vita della società umana e delle singole persone, e del loro rapporto con il mondo e con Dio Creatore e Provvidente. La natura umana richiede uno sviluppo inarrestabile del sapere, poiché la persona è agente di verità, per usare l'espressione del fenomenologo Robert Sokolowski ${ }^{5}$.

La vita umana richiede una incessante ricerca sotto molti aspetti. Le cosiddette scienze naturali o sperimentali, per ciò che riguarda la faccia empiricamente misurabile. E le scienze che cercano di conoscere ciò che viene

\footnotetext{
${ }^{3}$ FRANCESCO, Discorso nel $V$ convegno nazionale della chiesa italiana, Firenze, 10 novembre 2015.

${ }^{4}$ Ved. Francesco, Lettera al presidente della Pontificia Commissione per l'America Latina, del 19 marzo 2016. Una lettera particolarmente importante.

${ }^{5}$ Cfr. R. Sokolowski, Phenomenology of the Human Person, Cambridge University Press, 2008.
} 
osservato in altro modo e che fa capo allo spirito umano. L'impatto di Takashi Nagai davanti allo sguardo di amore di sua madre morente è un esempio chiaro di questo altro tipo di esperienza, ugualmente reale. Il linguaggio umano appartiene a questo ambito. È un linguaggio sintattico, che implica un salto rispetto alla comunicazione tra gli animali più perfetti. Non solo organismo biologico materiale, ma anche spirito, senza per ciò avere un dualismo. Quindi sono oggetto di ricerca il senso della vita, la dignità umana, la dualità uomo - donna, la famiglia, la verità, il dialogo, la cooperazione, l'amore, l'amicizia, la giustizia, e un lungo eccetera.

L'università ha subito una notevole trasformazione: non solo si è specializzata, ma si è frammentata. Non è un cambiamento così recente. Nelle conversazioni con alcuni dei partecipanti a un seminario nei primi anni ' 70 del secolo scorso attorno ad Augusto Del Noce, Cornelio Fabro e Carlos Cardona emergeva una forte consapevolezza del profondo mutamento culturale sul piano universitario e sociale, sotto vari aspetti: l'indebolimento della cultura politica, secondo Del Noce; la deriva ateistica dell'antropocentrismo immanentistico e il bisogno di un pensiero metafisico all'altezza delle sfide, per Fabro; il collegamento tra etica e metafisica, tra filosofia, teologia ed esistenza comune, in Cardona. Quest'ultimo esprimeva anche con lungimiranza la ricaduta, in atto da secoli, in ambito universitario dall'affrancamento di molte scienze rispetto alla sapienza filosofica e teologica. L'università era gravemente malatta ${ }^{6}$.

In un certo senso non si scopriva nulla di nuovo. John Henry Newman con la sua The Idea of a University (1852-1873) aveva affrontato il tema, creando lui stesso un'università a Dublino, forte della sua esperienza oxoniense. Quest'opera è rimasta una pietra miliare per pensare sempre di nuovo all'identità dell'istituzione e della vita universitaria, sottolineando che senza teologia non esiste università in senso prorio. Romano Guardini, Karl Jaspers, Ortega y Gasset e molti altri ci hanno lasciato un tesoro di considerazioni da rivisitare nelle attuali nuove circostanze.

$\mathrm{Ma}$ al tempo stesso negli anni '70 predevano forme più definite le successive trasformazioni dell'università e si potevano già scoprire le tendenze in atto di sviluppo futuro. Presso l'Università della Santa Croce, nata proprio un decennio dopo, ma ormai in questo periodo di cambiamenti più accellerati,

\footnotetext{
${ }^{6}$ In quel clima di lavorare per il futuro, cercando di arricchire la necessaria specializzazione scientifica con una riflessione sul posto di ogni sapere, J.J. Sanguineti scrisse uno studio importante dal punto di vista dei fondamenti: La filosofía de la ciencia según Santo Tomás, Eunsa, Pamplona 1977.
} 
la riflessione è stata presente fin dall'inizio, orientando le scelte di ricerca e di didattica, e anche, per quanto possibile negli aspetti strumentali. San Giovanni Paolo II e Benedetto XVI, con la loro esperienza di professori universitari, hanno scritto importanti discorsi rivolti a suoi colleghi in molte università del mondo, cercando di orientarne la loro crescita per il bene dell'umanità?.

La specializzazione è ulteriormente cresciuta in un clima culturale ,liquido" (Z. Baumann), senza punti di riferimento fermi. Da una parte, la maggior parte delle università più prestigiose sono diventate un insieme di centri di ricerca di eccellenza -termine questo da intendere piuttosto come servizio altamente qualificato alla società-, poco collegati tra di loro. Altri centri universitari invece sono sorti senza ricerca, quali scuole professionali vicine alla propria città, destinate semplicemente al training dei giovani per l'avviamento al lavoro, con docenti spesso di grande esperienza nella propria professione.

In entrambi i casi, domina in modo unilaterale l'aspetto di un servizio utile alla società nell'attuale congiuntura economica e lavorativa. Unilaterale, perché la società ha anche altre bisogni. In questo senso, non si possono leggere senza un timore salutare „Tre scritti sull'università" di Romano Guardini sulle responsabilità delle istituzioni universitarie. Tra questi scritti c'è un discorso agli studenti sulla loro responsabilità nei confronti della cultura, che oggi appare sorprendente; e la traccia per una conferenza dal significativo titolo „Volontà di potenza o volontà di verità?”.

La ricerca finalizzata a specifici bisogni di progresso, in modo particolare materiale, richiede dei grossi finanziamenti. Per cui, ogni volta di più le università dipendono da gruppi economici che sovvenzionano ricerche applicate in attesa di determinati risultati. In parte è giusto che sia così, ma ci sono anche gravi rischi di trasformare l'intera università in semplice strumento al servizio dello stato o di grandi gruppi nazionali o internazionali d'imprese. La società civile ha bisogno di questo progresso, ma anche di coesione, di fiducia, di senso, di responsabilità etica e di giustizia, di trascendenza religiosa, e come elemento basilare: la ricerca di verità nei diversi campi del reale.

\footnotetext{
${ }^{7}$ Questi discorsi hanno guidato la crescita dell'università. Cfr. dal punto di vista della Teologia Fondamentale, G. TANZElla-NitTI, Passione per la verità e responsabilità del sapere. Un'idea di università nel magistero di Giovanni Paolo II (1998). Poi I. Martínez-Echevarría sotto l'aspetto giuridico e filosofico,in La relación de la Iglesia con la Universidad en los discursos de Juan Pablo II y Benedicto XVI: una nueva aproximación jurídica (2010). Il $25^{\circ}$ anniversario dell'Università è stata un'occasione per riflettere insieme sui primi anni di percorso, come volume collettivo Pontificia Università della Santa Croce. Dono e compito: 25 anni di attività (2010).
} 
Da una diecina di anni, non si può parlare di trasformazione universitaria senza un riferimento al protocollo di Bologna. La globalizzazione nel campo economico e lavorativo richiede una omologazione dei titoli universitari a livello internazionale. Concretamente, l'Unione Europea ha messo in moto il cosiddetto processo di Bologna per ottenere un livello comune adeguato e garantito. Uno strumento importante di questo processo, non solo universitario, ma anche economico, politico e culturale, sono le agenzie per la valutazione e promozione della qualità delle università europee. Le accreditazioni richieste ai docenti e alle facoltà tendono a migliorare la qualità e l'efficacia della didattica, degli strumenti, delle risorse, ecc. Molti centri universitari hanno colto l'occasione di queste esigenze per fare dei progressi nel coordinamento dei programmi di studio, dei dipartimenti, dei modi di valutare il lavoro.

Si corre però anche il rischio che in un clima culturale dominato dalla tardomodernità, lo sforzo si concentra sugli aspetti organizzativi e procedurali. Infatti non è facile evitare il prevalere della ragione strumentale che concentrata più sui mezzi che sui fini. Sui pericoli che corriamo si possono leggere avvisi molto opportuni di W. von Humbolt, F. De Sanctis, M. Weber, F.A. von Hayek, G. Morra e molti altri. Mi limito a riportarne uno di H. Arendt in Sulla violenza, quando parla della necessità del distacco intellettuale e della ricerca disinteressata della verità, e a proposito delle università afferma che ,è improbabile che una qualche società civile possa soppravvivere alla scomparsa di queste curiose istituzioni la cui principale funzione politica e sociale si fonda precisamente sulla loro imparzialità e indipendenza dalle pressioni sociali e dal potere politico".

\section{LA FORMAZIONE DEL DOCENTE E RICERCATORE}

Un certo numero di università si pongono la questione di come educare le persone in un'università molto frammentata e massificata. Sorgono quindi degli interrogativi essenziali: che cos'è oggi un'università? come coltivare la specializzazione senza frammentare tutto il sapere? quale il ruolo dei saperi umanistici? è stato un bene isolare la teologia?

Alasdair MacIntyre si è occupato del tema per diversi anni en varie occasioni affermando che con la frammentazione del sapere, le università diventano multiversità ${ }^{8}$. Secondo lui, è necessario centrare l'impresa universitaria

\footnotetext{
${ }^{8}$ Catholic Universities. Dangers, Hopes, Choices, in: R.E. Sullivan (ed.), Higher Learning and Catholic Traditions, Notre Dame 2001; The End of Education. The Fragmentation of the
} 
nella "nozione della natura e dell'ordine delle cose, di un solo universo, i cui aspetti sono oggetto di ricerca di varie discipline, ma in modo tale che ogni aspetto necessita di essere messo in relazione con qualsiasi altro".

L'esperienza dice che l'interdisciplinarietà sia un cammino molto bello, ma anche che richiede una notevole apertura ed umiltà per accettare i limiti del proprio metodo. In primo luogo da parte degli scienziati per non chiudersi ai livelli non verificabili con il proprio metodo, per allargare la ragio$n e$, come ha indicato più volte Benedetto XVI. Ma anche da parte dei filosofi e dei teologi, per ascoltare sul serio e quindi imparare bene gli aspetti concreti della ricerca scientifica, con il linguaggio ad essa proprio.

MacIntyre propone di collocare al centro dell'università l'insegnamento e di affrontare specialmente un'antropologia integrale, con la collaborazione di scienziati in fisica, chimica, biologia, storia, economia, sociologia, oltre a filosofi e teologi. Questo progetto richiederebbe un lavoro in équipe di numerosi pensatori cattolici per un lungo periodo di tempo. Il suo centro integratore sarebbe una filosofía, elaborata ad alto livello accademico, e esenzialmente aperta alla teologia e alle scienze naturali e umane.

La sua proposta s'ispira in San Tommaso d'Aquino, con la sua distinzione di un duplice ordine di conoscenza -naturale e soprannaturale- e anche nell'idea di università" di John Henry Newman.

Dopo la rivoluzione antropologica del '68 che ha messo in questione convinzioni prima pacificamente condivise, si senta un forte bisogno di ripensare l'antropologia, partendo però dalle questioni che sono il compito di ogni Facoltà o Dipartimento universitario. La filosofia non dovrebbe essere un'illuminazione estrinseca. Infatti - perlomeno nello stile aristotelico sono le stesse questioni scientifiche ad esigere la riflessione dello scienziato. A questo punto il ricercatore - se ha il coraggio di pensare fino in fondo può sentire il bisogno di acquistare o di perfezionare l'abito intellettuale del ragionamento filosofico, studiando questi saperi e cercando il dialogo con coloro che hanno esercitato una vita intellettuale dedicata a questi abiti sapienziali intellettuali e morali.

Ma il passaggio al livello filosofico (e a quello teologico) è una collaborazione interdisciplinare dello stesso tipo delle precedenti? Non appare piuttosto come un qualche indagine verso l'interno e verso il fondamento?

American University, in „Commonweal”, October 20, 2006; God, Philosophy, Universities: A Selective History of the Catholic Philosophical Tradition, Rowman \& Littlefield Publishers Inc., Lanham, Maryland 2009, 173-180; The very Idea of a University: Aristotle, Newman, and us, in „British Journal of Educational Studies", vol. 57, no. 4, December 2009, pp. 347-362. 
Queste domande sono giustificate poiché infatti si passa da un sapere settoriale e particolare a un sapere pienamente universale o integrale, in qualche modo onnicomprensivo: sapere penultimo, la filosofia prima o metafisica, e sapere ultimo, la teologia.

„In modo più o meno consapevole, ogni scienza naturale presuppone una meta-fisica, una meta-psicologia, una meta-clinica, una meta-sociologia [...] In altre parole, fisica, psicologia, medicina e sociologia neanche saprebbero di che cosa parlano"9. Si può dire che il diritto suscita delle questioni metagiuridiche (filosofia del diritto) ${ }^{10}$ oppure che nella società esistono aspetti meta-politici. Questo meta-sapere, cioè l'esigenza di andare oltre -anzi andare nel profondo della realtà- vale per ogni scienza e per ogni professione, se non si vogliono ricevere i presupposti in modo acritico, senza esaminarli ${ }^{11}$.

Spesso non si vuole rimanere nei dati empirici, ma si opera con degli „strumenti” razionali o epistemologici poveri. Non sono molti gli scienziati che posseggono una sufficiente formazione filosofica, che permetta di riconoscere i limiti del sapere scientifico e, per tanto di evitare di andare impreparati al di là della propria competenza.

Questa sembra essere una possibile direzione di progresso: riconoscere che nella realtà esistono diversi livelli di perfezione a noi accessibili con metodi propri e adeguati. Questa distinzione di livelli d'intelligibilità dell'essere ed epistemologica dei metodi non lede l'unità molteplice e variegata del reale ${ }^{12}$.

Da secoli viene fatta una filosofia del diritto, con lo sviluppo delle questioni epistemologiche e dei temi meta-giuridici. La medicina ha studiato molto spesso la propria storia, alcune volte anche la filosofia della medicina e adesso non poche facoltà hanno un dipartimento di medical humanities. Oggi sembra importante collocare le singole scienze e le professioni che le applicano o le sviluppano, nella cornice della vita delle persone.

In sintesi, appaiono principalmente quattro compiti per una Facoltà universitaria al servizio dell'uomo integrale, che sono meta-scientifiche:

\footnotetext{
${ }^{9}$ Ved. G.B. Torello, El sentido último de la vida, según la „Fides et ratio” (1999), nel sito almudi.org.

${ }^{10}$ S. CotTA, Perché il diritto, Brescia $2^{\mathrm{a}}$ ed 1983; C.J. ERRÁzuriz, L'etica e il diritto: istanze separate o armoniche?, in „Iustitia”, 53 (2000), pp. 130-137.

${ }^{11}$ Cfr. Ll. Clavell, La metadisciplinarità. Scienza, filosofia e teologia, in E. MARIANI (a cura di), Quaderni dell'IPE, Napoli 2001, pp. 43-54.

${ }^{12}$ Cfr. J. MARITAIN, Distinguer pour unir: ou Les degrés du savoir, Desclée de Brouwer, Paris, 1932; M.A. VitoriA, El alcance cognoscitivo de la físico-matemática según Maritain, in „Acta Philosophica" 15 (2006/II), pp. 287-316.
} 
1) riflettere sui presupposti antropologici della scienza e professione;

2) essere consapevoli della questione epistemologica dei livelli di conoscenza;

3) studiare la dimensione etica della scienza e della professione;

4) ascoltare la parola di Dio rivelato in Cristo: teologia.

I primi tre punti sono compito delle scienze filosofiche, ma anche della letteratura (in modo universale) e della storia (in modo singolare).

Tommaso d'Aquino inizia la sua carriera accademica con un commento al libro De Trinitate de Boezio (sepolto a Pavia, come Agostino d'Ippona, entrambi con culto liturgico) per stabilire bene l'oggetto e il metodo della teologia nell'insieme di tutti i saperi. Ma finite le questioni $5^{\mathrm{a}}$ e $6^{\mathrm{a}}$, preso da tanti lavori, non finisce il suo commento. Più avanti scrisse con calma il suo commento alle opere di Aristotele e di nuovo tratta dello statuto delle diverse scienze. Sono molto illuminanti i proemi a questi scritti.

Jean-Pierre Torrell racconta un esempio della serietà professionale di Tommaso: quando elabora lo studio delle virtù nella seconda parte della Summa Teologica sente il bisogno di studiare con profondità l'Etica Nicomachea, scrivendo il suo commento, non per l'insegnamento, ma per assimilare un etica naturale particolarmente profonda.

Come diceva Augusto del Noce, viviamo in un mundo pieno di frammenti filosofici, presenti nel mondo della comunicazione. La sapienza filosofica e teologica non è un lusso ma una necessità per poter dialogare seriamente, per lasciare che la mente legga nella realtà (intus-legere), per essere aperti nella ricerca in vista di conoscere meglio la verità. Da questo punto di vista, sembra esemplare il lavoro di Robert Spaemann, morto recentemente, sulla finalità iscritta in tutto il reale ${ }^{13}$; stanno insieme la prospettiva storica, la filosofia e la scienza nei loro rapporti reciproci. Così si facilita il dialogo, e si possono smascherare i dualismi presenti nella cultura contemporanea. Dualismi natura-cultura, natura-libertà, natura-ragione, che ostacolano la conoscenza del reale.

Chiudo questo articolo con due ricordi. Le parole di Edmund Husserl nella conferenza La crisi dell'umanità europea e la filosofia del 7 maggio 1935 a Vienna: „La crisi dell'esistenza europea ha solo due sbocchi: il tramonto dell'Europa, nell'estraneazione rispetto al senso razionale della

\footnotetext{
${ }^{13}$ Fini naturali: storia \& riscoperta del pensiero teleologico (prefazione del card. Camillo Ruini traduzione a cura di Leonardo Allodi e Giacomo Miranda, Ares, Milano 2013) tiene insieme la prospettiva storica, la filosofia e la scienza nei loro rapporti reciproci. Così anche in altre sue opere (ad es. R. Spaemann, Natura e ragione: saggi di antropologia, presentazione di Luca F. Tuninetti, EDUSC, Roma 2006.
} 
propria vita, la caduta nell'ostilità allo spirito e nella barbarie, oppure la rinascita dell'Europa dallo spirito della filosofia, attraverso un eroismo della ragione capace di superare definitivamente il naturalismo. Il maggior pericolo dell'Europa è la stanchezza"14.

Il secondo ricordo è più personale. Il 10 giugno 1996, alcuni professori siamo stati ricevuti da san Giovanni Paolo II in un'udienza poco numerosa, molto affettuosa e un po' informale. Nella conversazione, parlando della situazione attuale e dell'attrazione di molti giovani verso la filosofia, il Papa ha detto con convinzione in latino: metaphysica utilis ad omnia. Poi ha spiegato che era un'espressione che un suo professore di filosofia e di teologia ripeteva spesso. Per me era una conferma di quanto avevo compreso studiando i suoi più importanti discorsi sulla figura di san Tommaso d'Aquino lungo il suo pontificato. In essi infatti è sempre presente la metafisica, accanto ai caratteristici aspetti antropologici. Si può dire che Wojtyla ha arricchito la metafisica con una base esperienziale antropologica più ampia. Penso quindi che la sua fenomenologia non è intesa come metodo di fondazione ultima, anche se necessario per cogliere il soggetto in quanto tale. Se non erro, era la realizzazione della profonda visione di Étienne Gilson secondo la quale: „la vraie métaphysique de l'être n'a jamais eu la phénoménologie à laquelle elle avait droit, la phénoménologie moderne n'a pas la métaphysique qui peut seule la fonder, et, en la fondant, la guider" ${ }^{\prime 15}$.

\section{CENNI BIBLIOGRAFII}

Ashley B., The Way toward Wisdom: An Interdisciplinary and Intercultural Introduction to Metaphysics, Notre Dame, Ind.: University of Notre Dame Press 2006.

ARANDA A., (ed.), Identidad cristiana. Coloquios universitarios (2007). Raccoglie colloqui universitari sull'identità cristiana con la partecipazione di docenti di varie facoltà e quindi da molteplici angoli del sapere: filosofia, teologia, diritto, comunicazione, medicina, scienza, storia, economia ed etica.

Llano A., Repensar la Universidad. La Universidad ante lo nuevo (2003).

Lombo J.A., Gimenez AmAya J.M., La unidad de la persona: aproximación interdisciplinar desde la filosofía y la neurociencia, Pamplona: EUNSA, 2013.

\footnotetext{
${ }^{14}$ Ringrazio che G. Morra, abbia volute ricordarle nelle sue riflessioni Università come Europa (2010), scritte per il volume in suo onore in occasione del suo $80^{\circ}$ genetliaco (a cura di Leonardo Allodi, Dove va la sociologia oggi?), pensando ai tanti anni di docenza e ricerca proprio nell'Università di Bologna.

${ }^{15}$ É. GILSON, L'être et l'essence, Librairie Philosophique, J. Vrin, Paris 1981, p. 22.
} 
MacIntyre A., Three Rival Versions of Moral Enquiry, The Gifford Lectures, University of Notre Dame Press, 1990.

McGrath A.E., Inventing the Universe. Why We Can't Stop Talking About Science, Faith, and God (2015).

- „Acta Philosophica”, 2013, vol. 22, fascicolo 2.

Quaderno monografico: The Idea of a University

- Lluís Clavell, Trasformazione delle università ed educazione. Presentazione del Quaderno monografico, pp. 211-216.

- José M. Giménez Amaya, Diagnóstico de la Universidad: un paradigma de la fragmentación (omni)presente. Apuntes para una reconstrucción, pp. 217-234.

- Reinhard HÜTTER, University Education, the Unity of Knowledge - and (Natural) Theology: John Henry Newman's Prophetic Provocation, pp. 235-256.

- José M. Torralba, La educación liberal como misión de la universidad. Introducción bibliográfica al debate sobre la identidad de la universidad, pp. 257-276.

\section{SOME PUBLICATIONS}

- Metafisica (in collaborazione con M. Pérez de Laborda), Ed. Università S. Croce, Roma 2006, pp. 365.

- Metafisica (in collaborazione con T. Melendo e T. Alvira), Ediciones Universidad de Navarra, $8^{\text {a }}$ ed.ampliata, Eunsa, Pamplona 2001, pp. 290 (reprint: 2007); Metaphysics (trad.inglese), Sinag-Tala Publ. Manila 1991; Metafisica (trad. italiana), Le Monnier, Firenze 1987, pp. 232.

- Philosophy and Sacred Text: Mutual Hermenutical Help. The Case of Ex 3:14, in P. RosZAK and J. Vijgen (Ed.), Reading Sacred Scripture with Thomas Aquinas. Hermeneutic tools, theological questions and new perspectives, Brepols 2015, pp. 457-479.

- Tommaso d'Aquino \& Robert Spaemann. Riscoprire la finalità naturale, in „Studi Cattolici” 2015, pp. 92-98.

- A New Impulse to Evangelization: The Reform of Philosophical Studies in Harmony with Theology, in ,Josephinum. Journal of Theology”, 2013, vol. 20, pp. 113-128.

- Trasformazione delle università ed educazione, presentazione del quaderno monografico „The Idea of a University” (essays of Reinhard Hütter, José Manuel Giménez Amaya, José M. Torralba), in „Acta Philosophica”, 2013, pp. 211-216.

- La collaboration entre foi et raison dans les sciences et dans la vie professionelle, in Philippe CAPelle-Dumont (Ed.), Confiance dans la raison, Parole et Silence, Paris 2012, pp. 243-259.

- Razón y fe en la universidad: ¿oposición o colaboración?, CEU Ediciones, Madrid 2010.

- Raccogliere l'eredità di Giovanni Paolo II su San Tommaso d'Aquino, in Doctor Communis 2006, pp. 21-48.

- Le dimensioni della libertà, in G. Borgonovo, K. Charamsa (Ed.), Eucaristia e Libertà, L. Ed. Vaticana, 2006, pp. 137-151.

- Trabajo y familia: superar la fragmentación partiendo de la persona, in Domènec MELE (Ed.), Conciliar trabajo y familia: un reto para el siglo XXI, EUNSA, Pamplona 2004, pp. 103-116. 
- Para superar la fragmentación del saber, in T. TRigo (Ed.) Dar razón de la esperanza. Homenaje al Prof. Dr. José Luis Illanes, Servicio de Publicaciones de la Universidad de Navarra, Pamplona 2004, pp.1149-1160.

- The Freedom Won by Christ on the Cross. Theological Reflections on Freedom in Blessed Josemaria's Teaching, in „Romana Bulletin of the Prelature of the Holy Cross and Opus Dei”, (English edition) 33(2001), pp. 234-261.

- Dottrina Sociale della Chiesa e Metafisica, in „La Società”, gennaio-marzo1999, pp. 121-127. L'articolo tradotto in polacco è apparso con il titolo Nauka spoleczna Kościoła i metafizyka, in ,Spoleczenstwo”, marzo 1999, pp. 135-142.

\section{UNIWERSYTET W SŁUŻBIE CZŁOWIEKA INTEGRALNEGO}

\section{STRESZCZENIE}

Autor rozpoczyna artykuł podaniem dwóch przykładów na temat pracy zawodowej wykonywanej z doskonałym przygotowaniem i umożliwiającej świadczenie pomocy człowiekowi w jego wymiarze personalnym i społecznym. Opowiada się za modelem uniwersytetu, który zdobywa i przekazuje wiedzę w służbie człowiekowi i społeczeństwu. Temu winna służyć współpraca różnych dyscyplin naukowych w uniwersytecie, a przygotowanie kadry naukowej winno być ukierunkowane na rzetelne badania naukowe i właściwy przekaz dydaktyczny.

Słowa kluczowe: Uniwersytet; badania naukowe; dydaktyka; służba; człowiek integralny. 